\title{
Determination of the laplace transform for the first falling moment to zero level of a semi-Markov random process
}

\author{
Selahattin Maden ${ }^{1}$ and Ulviyya Y. Karimova ${ }^{2}$ \\ ${ }^{1}$ Department of Mathematics, Faculty of Arts and Sciences, Ordu University, Ordu, Turkey \\ ${ }^{2}$ Department of Applied Mathematics and Cybernetics, Baku State University, Baku, Azerbaijan
}

Received: 8 September 2017, Accepted: 16 October 2017

Published online: 8 November 2017.

\begin{abstract}
One of the important problems of stochastic process theory is to define the Laplace transformations for the distribution of this process. With this purpose, we will investigate a semi-Markov random processes with positive tendency and negative jump in this article. The first falling moment into the zero-level of this process is constructed as mathematically and the Laplace transformation of this random variable is obtained.
\end{abstract}

Keywords: Semi-Markov process, Laplace transform, Erlang distribution.

\section{Introduction}

In recent years, random walks with one or two barriers are used to solve a number of very interesting problems in the fields of inventory, queues and reliability theories, mathematical biology etc. Many good monographs in this field exist in literature (see references [1]-[4] and etc.).

In particular, a number of very interesting problems of stock control, queues and reliability theories can be expressed by means of random walks with two barriers. These barriers can be reflecting, delaying, absorbing, elastic, etc., depending on concrete problems at hand. For instance, it is possible to express random levels of stock in a warehouse with finite volumes or queueing systems with finite waiting time or sojourn time by means of random walks with two delaying barriers. Furthermore, the functioning of stochastics systems with spare equipment can be given by random walks with two barriers, one of them is delaying and the other one is any type barrier.

Numerous studies have been done about step processes of semi-Markovian random walk with two barriers of their

practical and theoretical importance. But in the most of these studies the distribution of the process has free distribution. Therefore, the obtained results in this case are cumbersome and they will not be useful for applications ([1], [2], [3], [4] and etc.).

The investigations of the distributions for the processes of semi-Markov random process have an important value in the random process theory. There are number of works devoted to definition of the Laplace transforms for the distribution of the first passage of the zero level. Some authors used the asymptotic, factorization and etc. methods (see references [1], [2] and [5]). But other authors narrowing the class of distributions of walking found the evident form for Laplace transforms for distributions and its main characteristics (see [3], [4]). 
The purpose of the present article is to find the Laplace transforms for Erlang distribution of the semi-Markov random processes with positive tendency and negative jump. The first passage of the zero level of the semi-Markov process with positive tendency and negative jumps will be included as a random variable. The Laplace transform for the distribution of this random variable is defined.

\section{Construction of the process}

Suppose that $\left\{\left(\xi_{i}, \eta_{i}\right)\right\}, i=1,2,3, \ldots$ is a sequence of identically and independently distributed pairs of random variables, defined on any probability space such that $\xi_{i}$ 's are positive valued, i.e., $P\left\{\xi_{i}>0\right\}=1, i=1,2,3, \ldots$ In addition, the random variables $\xi_{i}$ and $\eta_{i}$ are mutually independent as well. Also let us denote the distribution function of $\xi_{1}$ and $\eta_{1}$

$$
\Phi(t)=P\left\{\xi_{1}<t\right\}, F(x)=P\left\{\eta_{1}<x\right\}, t \in R^{+}, x \in R
$$

respectively. By the means of these random variables, we can construct the following process:

$$
X(t)=z+t-\sum_{i=1}^{k-1} \eta_{i}, \text { if } \sum_{i=1}^{k-1} \xi_{i} \leq t<\sum_{i=1}^{k} \xi_{i}, k=(1, \infty)
$$

where the number $z>0$ is given. $X(t)$ process is a (asymptotic) semi-Markov random processes with positive tendency and negative jumps. One of the realizations of the process $X(t)$ will be in the following form

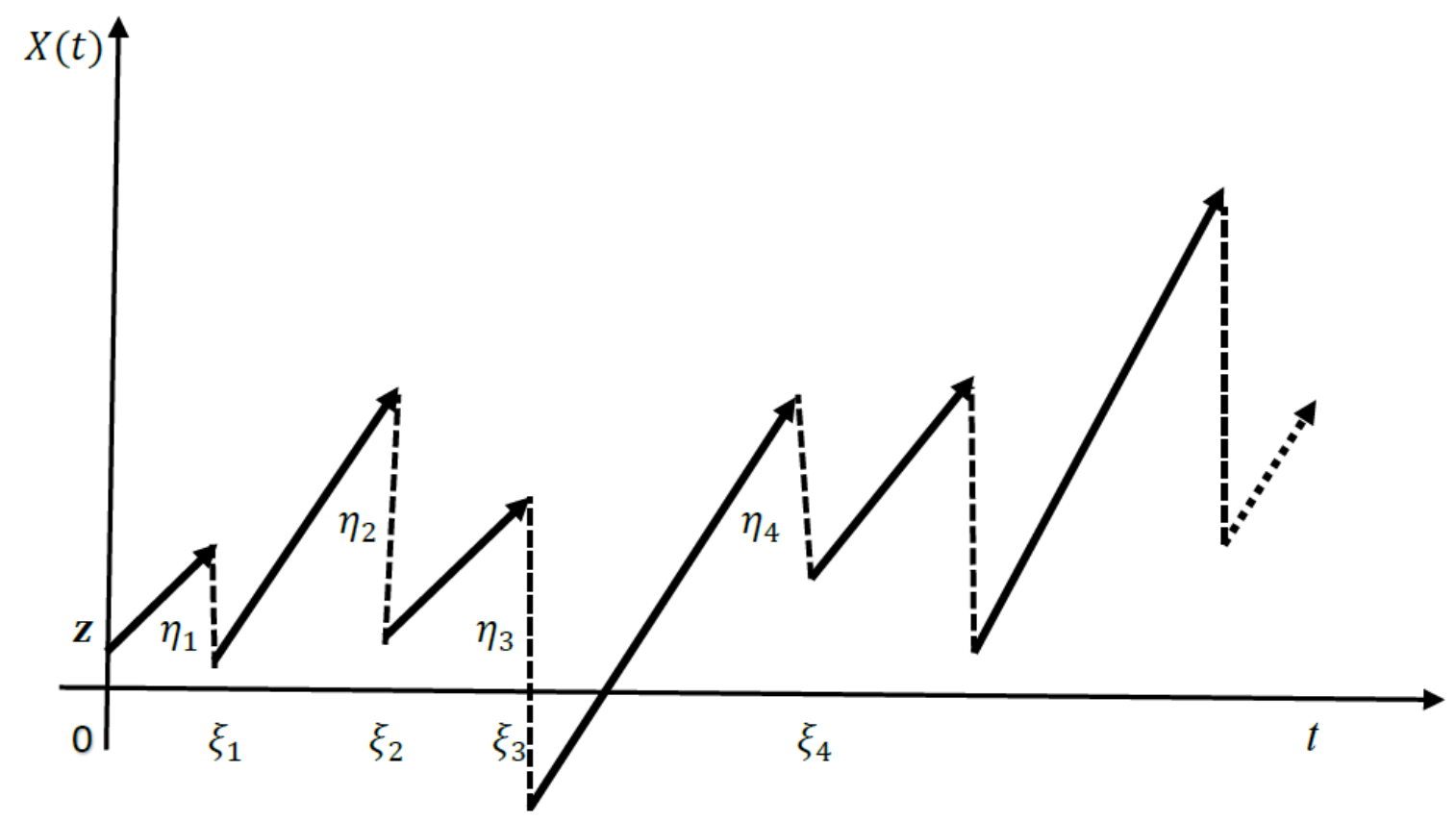

Fig. 1: A View of the Semi-Markov Random Process with positive tendency and negative jumps.

Now, we introduce the random variable as below.

$$
\tau_{0}=\tau_{0}(w)=\inf \{t: X(t) \leqslant 0\}
$$


where $\tau_{0}$ is the first falling time to the zero-level of the process $X(t)$. Furthermore, we get $\tau_{0}=+\infty$ when $X(t)>0$ for every $t$. It is obvious that the random variable $\tau_{0}$ is the time of the first crossing of the the process $X(t)$ into the zero level. In this case $\tau_{0}$ is rewritten as $\tau_{0}=\min \{k \geq 1: X(t)=0\}$. Thus, for example, we can write

$$
\tau_{0}=\left\{\begin{array}{c}
\xi_{1}, z+\xi_{1}-\eta_{1}<0 \\
\xi_{1}+T, z+\xi_{1}-\eta_{1}>0
\end{array}\right.
$$

where $\xi_{1}$ and $T$ are identically and independently distributed random variables.

Note that $\tau_{0}$ is important from a scientific and practical point of view and it is an important boundary functional of the process $X(t)$. This random variable play an important role in solving of most probability problems arising in control of random levels of stocks in a warehouse which is functioning according to the process $X(t)$. For this reason, the consideration with detailed of random variable $\tau_{0}$ seems very interesting from scientific and practical point of view.

The aim of the present work is to determine the Laplace transform of the distribution function of the random variable $\tau_{0}$. Let us denote the Laplace transform of the distribution function of the random variable $\tau_{0}$ by

$$
L(\theta)=E\left[e^{\left(-\theta \tau_{0}\right)}\right], \theta>0,(4)
$$

and let us denote the Laplace transform of the conditional distribution of the random variable $\tau_{0}$ by

$$
L(\theta \mid z)=E\left[e^{-\theta \tau_{0}} \mid X(0)=z\right], \theta>0, z \geq 0
$$

According to the total probability formula, we can write

$$
L(\theta)=\int_{z=0}^{\infty} L(\theta \mid z) d P(X(0)<z)
$$

Finally, let us denote the Laplace transformation of the random variable $\xi_{1}$ by

$$
\varphi(\theta)=E\left[e^{-\theta \xi_{1}}\right]
$$

\section{Derivation of the Integral Equation for $L(\theta \mid z)$}

In this section we give an integral equation for the Laplace transform of the conditional distribution of the random variable $\tau_{0}$. According to the formula of total probability, we have

$$
\begin{aligned}
L(\theta \mid z) & =E\left[e^{-\theta \tau_{0}} \mid X(0)=z\right]=\int_{\Omega} e^{-\theta t} P\{\mathrm{dw} \mid X(0)=z\} \\
& =\int_{\left\{\mathrm{w}: \mathrm{z}+\xi_{1}-\eta_{1}<0\right\}} e^{-\theta \xi_{1}} P\{\mathrm{dw}\}+\int_{\left\{\mathrm{w}: \mathrm{z}+\xi_{1}-\eta_{1}>0\right\}} e^{-\theta\left(\xi_{1}+T\right)} P\{\mathrm{dw}\}
\end{aligned}
$$


Applying some substitutions, we obtain that

$$
\begin{aligned}
E\left[e^{-\theta \tau_{0}} \mid X(0)=z\right] & =\int_{s=0}^{\infty} \int_{y=\mathrm{z}+\mathrm{s}}^{\infty} e^{-\theta s} P\left\{\xi_{1} \in d s ; \eta_{1} \in d y\right\}+\int_{s=0}^{\infty} \int_{y=0}^{\mathrm{z}+\mathrm{s}} \int_{\beta=0}^{\infty} e^{-\theta(s+\beta)} P\left\{\xi_{1} \in d s ; \eta_{1} \in d y ; T \in d \beta\right\} \\
& =\int_{s=0}^{\infty} e^{-\theta s} P\left\{\xi_{1} \in d s\right\} \int_{y=\mathrm{z}+\mathrm{s}}^{\infty} P\left\{\eta_{1} \in d y\right\}+\int_{s=0}^{\infty} e^{-\theta s} \int_{y=0}^{\mathrm{z}+\mathrm{s}} d P\left\{\eta_{1}<y\right\} d P\left\{\xi_{1}<s\right\} L(\theta \mid z+s-y) \\
& =\int_{s=0}^{\infty} e^{-\theta s} P\left\{\xi_{1} \in d s\right\} P\left\{\eta_{1}>z+s\right\}+\int_{s=0}^{\infty} e^{-\theta s} \int_{\beta=\mathrm{z}+\mathrm{s}}^{0} L(\theta \mid \beta) d P\left\{\eta_{1}<\mathrm{z}+\mathrm{s}-\beta\right\} d P\left\{\xi_{1}<s\right\}
\end{aligned}
$$

or, equivalently,

$$
L(\theta \mid z)=\int_{s=0}^{\infty} e^{-\theta s} P\left\{\xi_{1} \in d s\right\} P\left\{\eta_{1}>z+s\right\}+\int_{s=0}^{\infty} e^{-\theta s} \int_{y=0}^{\mathrm{z}+\mathrm{s}} L(\theta \mid z+s-y) P\left\{\eta_{1} \in \mathrm{dy}\right\} P\left\{\xi_{1} \in \mathrm{d} s\right\} .
$$

By substituting $z+s-y=\alpha$, we get the integral equation for $L(\theta \mid z)$.

$$
L(\theta \mid z)=\int_{s=0}^{\infty} e^{-\theta s} P\left\{\xi_{1} \in d s\right\} P\left\{\eta_{1}>z+s\right\}+\int_{s=0}^{\infty} e^{-\theta s} \int_{y=0}^{\mathrm{z}+\mathrm{s}} L(\theta \mid \alpha) P\left\{\eta_{1}<z+s-y\right\} d P\left\{\xi_{1}<s\right\}
$$

\section{The solution of the Integral Equation for $L(\theta \mid z)$}

The integral equation given in (6) for $L(\theta \mid z)$ can be solved by method of successive approximations for arbitrarily distributed random variables $\xi_{i}$ and $\eta_{i}, i \geq 1$, but it is unsuitable for applications. On the other hand, this equation has a solution in explicit form in the classes of Erlang distributions. Therefore, this integral equation will be solved in the cases that the random variables $\xi_{1}$ and $\eta_{1}$ have an Erlang distribution of second order with the parameter $\lambda$ and first order with the parameter $\mu$, respectively. In this case, we have

$$
\begin{aligned}
& P\left\{\xi_{1}(\omega)<t\right\}=\left[1-(1+\lambda \mathrm{t}) e^{-\lambda t}\right] \varepsilon(t), \lambda>0, \\
& \mathrm{P}\left\{\zeta_{1}(\omega)<t\right\}=\left[1-e^{-\mu t}\right] \varepsilon(t), \mu>0
\end{aligned}
$$

where

$$
\varepsilon(t)=\left\{\begin{array}{l}
0, \mathrm{t}<0 \\
1, \mathrm{t}>0
\end{array}\right.
$$

Thus, according to the total probability formula, we have the following integral equation for the Laplace transform of the conditional distribution of random variable $\tau_{0}$

$$
L(\theta \mid z)=\frac{\lambda^{2} e^{-\mu \mathrm{z}}}{(\lambda+\mu+\theta)^{2}}-\lambda^{2} \mu e^{-\mu \mathrm{z}} \int_{s=0}^{\infty} s e^{-(\lambda+\mu+\theta) \mathrm{s}} \int_{\alpha=0}^{z+s} e^{\mu \alpha} L(\theta \mid \alpha) d \alpha \mathrm{ds}
$$

Now, we will receive a differential equation. For this aim, we will multiply both sides of equation (7) by $e^{\lambda z}$ and derive on $z$.

$$
\mu \mathrm{L}(\theta \mid \mathrm{z})+\mathrm{L}^{\prime}(\theta \mid \mathrm{z})=\lambda^{2} \mu \int_{\mathrm{s}=0}^{\infty} s e^{-(\lambda+\theta) \mathrm{s}} \mathrm{L}(\theta \mid \mathrm{z}+\mathrm{s}) \mathrm{ds}
$$


Then we will multiply both sides of last equation by $e^{-(\lambda+\mu+\theta) z}$ and derive on $z$. In this case we will receive the differential equation with 3-th construction

$$
L^{\prime \prime \prime}(\theta \mid z)-[2(\lambda+\theta)-\mu] L^{\prime \prime}(\theta \mid z)+(\lambda+\theta)(\lambda+\theta-2 \mu) L^{\prime}(\theta \mid z)+(2 \lambda+\theta) \mu \theta \mathrm{L}(\theta \mid \mathrm{z})=0
$$

The general solution of integral equation (7) has the form

$$
L(\theta \mid z)=C_{1}(\theta) \mathrm{e}^{\mathrm{k}_{1}(\theta) \mathrm{z}}+C_{2}(\theta) \mathrm{e}^{\mathrm{k}_{2}(\theta) \mathrm{z}}+C_{3}(\theta) \mathrm{e}^{\mathrm{k}_{3}(\theta) \mathrm{z}}
$$

where $k_{i}(\theta), i=1,2,3$ are the roots of characteristic equation of

$$
k^{3}(u)-[2(\lambda+\theta)-\mu] k^{2}(u)+(\lambda+\theta)(\lambda+\theta-2 \mu) k(u)+(2 \lambda+\theta) \mu \theta \mathrm{L}(\theta \mid \mathrm{z})=0
$$

By finding $C_{i}(\theta), \mathrm{i}=1,2,3$ from equation (6) we will get the following system of algebraic equations.

$$
\left\{\begin{array}{l}
L(\theta \mid 0)=\frac{\lambda^{2}}{(\lambda+\mu+\theta)^{2}}+\lambda^{2} \mu \int_{\mathrm{s}=0}^{\infty} \mathrm{s} \mathrm{e}^{-(\lambda+\mu+\theta) \mathrm{s}} \int_{\alpha=0}^{0} e^{\mu \alpha} d \alpha d s \\
\mathrm{~L}^{\prime}(\theta \mid 0)=-\mu \mathrm{L}(\theta \mid 0)+\lambda^{2} \mu \int_{\mathrm{s}=0}^{\infty} \mathrm{s}^{2} \mathrm{e}^{-(\lambda+\theta) \mathrm{s}} \mathrm{L}(\theta \mid \mathrm{s}) \mathrm{ds} \\
L^{\prime \prime}(\theta \mid 0)=\mu(\lambda+\theta) \mathrm{L}(\theta \mid 0)+(\lambda+\theta-\mu) \mathrm{L}^{\prime}(\theta \mid 0)-\lambda^{2} \mu \int_{\mathrm{s}=0}^{\infty} \mathrm{e}^{-(\lambda+\theta) \mathrm{s}} \mathrm{L}(\theta \mid \mathrm{s}) \mathrm{ds}
\end{array}\right.
$$

From these conditions, taking into account expression (6), we obtain the following system of equations with respect to $C_{i}(\theta)$,

$$
\left\{\begin{array}{l}
\sum_{i=1}^{3}\left[1-\frac{\lambda^{2} \mu\left[2(\lambda+\theta)+\mu-k_{i}(\theta)\right]}{\left[\lambda+\theta-k_{i}(\theta)\right]^{2}[\lambda+\mu+\theta]^{2}}\right] \mathrm{C}_{\mathrm{i}}(\theta)=\frac{\lambda^{2}}{(\lambda+\mu+\theta)^{2}} \\
\sum_{i=1}^{3}\left[\mu+k_{i}(\theta)-\frac{\lambda \mu}{\lambda+\theta-k_{i}(\theta)}\right] \mathrm{C}_{\mathrm{i}}(\theta)=0 \\
\sum_{i=1}^{3}\left[k_{i}^{2}(\theta)-(\lambda+\theta-\mu) \mathrm{k}_{\mathrm{i}}(\theta)-\mu(\lambda+\theta)+\frac{\lambda^{2} \mu}{\lambda+\theta-k_{i}(\theta)}\right] \mathrm{C}_{\mathrm{i}}(\theta)=0
\end{array}\right.
$$

Now we will proof linear dependence of this algebraic system. In this case, if we consider the following equalities

$$
\begin{aligned}
& k_{1}(\theta)+k_{2}(\theta)+k_{3}(\theta)=(\lambda+\theta)-\mu \\
& k_{1}(\theta) k_{2}(\theta)+k_{1}(\theta) k_{3}(\theta)+k_{2}(\theta) k_{3}(\theta)=2(\lambda+\theta)(\lambda+\theta-\mu)
\end{aligned}
$$

and

$$
k_{1}(\theta) k_{2}(\theta) k_{3}(\theta)=(\lambda+\theta)(\lambda+\theta-2 \mu)
$$

then we can write

$$
\left[\mu+k_{1}(\theta)\right]\left[\mu+k_{2}(\theta)\right]\left[\mu+k_{3}(\theta)\right]=\lambda^{2} \mu .
$$


Therefore, after some transformations, the boundary conditions given the equation (11) take the following form

$$
\left\{\begin{array}{l}
\sum_{i=1}^{3}\left(\lambda-k_{i}(\theta)+\theta\right)^{2} \mathrm{C}_{\mathrm{i}}(\theta)=\lambda^{2} \\
\sum_{i=1}^{3} 0 \times C_{i}(\theta)=0 \\
\sum_{i=1}^{3} 0 \times C_{i}(\theta)=0
\end{array}\right.
$$

Thus, the system (11) is a linear dependent equations system, as

$$
\begin{aligned}
& C_{2}(\theta)=C_{3}(\theta)=0, \theta>0, \\
& C_{1}(\theta)=\frac{\lambda^{2}}{\left[\lambda+\theta-k_{1}(\theta)\right]^{2}} .
\end{aligned}
$$

So, the general solution of integral equation (6) will be as follows. This is the Laplace transform for relative distribution of random variable $\tau_{0}$.

Since random variable has a first order Erlang distribution with the parameter $\mu$, the Laplace transform for non-relative distribution of random variable $\tau_{0}$ will be

$$
\begin{aligned}
L(\theta) & =\int_{z=0}^{\infty} L(\theta \mid z) \lambda^{2} \mathrm{ze}^{-\lambda \mathrm{z}} d z=\int_{z=0}^{\infty} C_{1}(\theta) e^{k_{1}(\theta) z} \lambda^{2} z \mathrm{e}^{-\lambda z} d z \\
& =C_{1}(\theta) \lambda^{2} \int_{z=0}^{\infty} z e^{\left[k_{1}(\theta-\lambda] z\right.} d z=\frac{\lambda^{2}}{\left[\lambda-k_{1}(\theta)\right]^{2}} C_{1}(\theta)
\end{aligned}
$$

We now determine $E\left[\tau_{0}\right]$ and $D\left[\tau_{0}\right]$, expectation and standart diversion of the random variable $\tau_{0}$. Since is a Laplace transformation, it is written

$$
E\left[\tau_{0}\right]=-L^{\prime}(0) \text { and } D\left[\tau_{0}\right]=-L^{\prime \prime}(0)-\left[L^{\prime}(0)\right]^{2}
$$

Therefore, we can write expectation and standard diversion of random variable $\tau_{0}$ for $\lambda>2 \mu$, as follows.

$$
\begin{aligned}
& E\left[\tau_{0}\right]=-L^{\prime}(0)=\frac{2(\lambda+\mu)}{\lambda(\lambda-2 \mu)} \\
& E\left[\tau_{0} \mid z\right]=\frac{2(1+z \mu)}{\lambda-2 \mu}
\end{aligned}
$$

and

$$
\begin{aligned}
& L^{\prime \prime}(0)=\frac{8 \mu^{2}}{\lambda^{2}(\lambda-2 \mu)^{2}}+\frac{2 \mu}{\lambda(\lambda-\mu)(\lambda-2 \mu)}+\frac{2 \lambda}{(\lambda-2 \mu)^{3}} \\
& D\left[\tau_{0}\right]=\frac{8 \mu^{2}}{\lambda^{2}(\lambda-2 \mu)^{2}}+\frac{16 \mu}{\lambda(\lambda-\mu)(\lambda-2 \mu)}+\frac{3 \lambda}{(\lambda-2 \mu)^{3}}-\frac{4(\lambda+2 \mu) \mu}{\lambda^{2}(\lambda-2 \mu)^{2}} \\
& D\left[\tau_{0} \mid z\right]=\frac{2}{(\lambda-2 \mu)^{2}}+\frac{(2+z \lambda) \mu}{(\lambda-2 \mu)^{3}}
\end{aligned}
$$




\section{Conclusion}

In this article we have defined Laplace transforms for Erlang distribution of the first falling time to zero level of semiMarkov random process with positive tendency and negative jump. The obtained results can be applied in the theory of queuing, in insurance theory, in the theory of finance, and also in the theory of inventory management.

\section{Competing interests}

The authors declare that they have no competing interests.

\section{Authors' contributions}

All authors have contributed to all parts of the article. All authors read and approved the final manuscript.

\section{References}

[1] Afanas'eva, L. G., Bulinskaya, E. V., 'Some asymptotical results for random walks in a strip' Teor. Veroyatn. Primen. 29, 4, 658-668 (1983).

[2] Borovkov, A. A., Stochastic Processes in The Theory of Queues, Nauka, Moscow 1972.

[3] Borovkov A. A., 'On the asymptotic behaviour of the distributions of the first passage', Mat. Zametki, Vol.75, No.1, pp.24-39, (2004).

[4] Feller, W., An Introduction to Probability Theory and Its Applications, Vol. I, Wiley, New York 1968.

[5] Gihman, I. I., Skorohod, A. V., The Theory of Stochastic Processes II, Springer-Verlag, 1975.

[6] Lotov, V. I., On some boundary crossing problems for gaussian random walks, The Annals of Probab., 24, 1996, pp.2154-2171, (1996).

[7] Lotov, V. I., 'On the asymptotics of distributions in two-sided boundary problems for random walks defined on a Markov chain', Sib. Adv. Math. 1, 3, 26-51 (1991).

[8] Nasirova, T. H, Karimova ,U. Y., 'Definition of Laplace transform of the first passage of zero level of the semimarkov random process with positive tendency and negative jump2, Applied mathematics, 2, pp. 908-912, (2011).

[9] Nasirova, T. I., and Shamilova, B. G., 'Investigation of Some Probabilistic Characteristics of One Class of Semi Markov Wandering with Delaying Screens', Automatic Control and Computer Sciences, Vol. 48, No. 2, 109-119 (2014).

[10] Nasirova, T. I., Sadikova, R. I., and Ibaev, E. A., 'Determination of the Mean and Mean Square Deviations of the System Level', Automatic Control and Computer Sciences, Vol. 49, No. 1, 37-45 (2015).

[11] Omarova, K. K., Bakhshiev, S. B., 'The Laplace Transform for the Distribution of the Lower Bound Functional in a Semi Markov Walk Process with a Delay Screen at Zero', Automatic Control and Computer Sciences, Vol. 44, No. 4, 246-252 (2010).

[12] Omarova, K. K., 'Laplace transformation of ergodic distribution of the step process of semi-markov random walk with delaying screen at positive point', The Third International Conference "Problems of Cybernetics and Informatics", (2010).

[13] Prabhu, N. U., Stochastic Storage Processes, New York, Springer-Verlag, 1981.

[14] Spitzer, F., Principles of Random Walk, Van Nostrand, Princeton, 1969. 